M. Manaresi

Nagoya Math. J.

Vol. 77 (1980), 61-74

\title{
SOME PROPERTIES OF WEAKLY NORMAL VARIETIES*)
}

\author{
MIRELLA MANARESI
}

\section{Introduction}

In this paper we give a new characterization of weak normalization and we use it to discuss some questions about weakly normal varieties formulated by A. Andreotti and E. Bombieri in [1] and studied by S. Greco and C. Traverso in [8] for seminormality.

The results we obtain are similar to the ones of Greco and Traverso, but they are based on a base-change theorem (see: Theorem (III. 1)) which is proved by standard results of commutative algebra not involving Traverso's gluings (used in [8]).

Since the questions are local, we limit ourselves to affine schemes (i.e. to rings), and we use the language of [9] and [13].

In section $I$ we prove that if $A$ is a ring, $B$ an overring of $A$ integral over $A$, the weak normalization of $A$ in $B$ is the subring ${ }_{B}^{*} A$ of $B$ such that the sequence

$$
{ }_{B}^{*} A \longrightarrow B \underset{g}{\stackrel{f}{\longrightarrow}}\left(B \otimes_{A} B\right)_{\mathrm{red}}
$$

is exact, where $f, g$ are the ring homomorphisms

$$
\begin{array}{ll}
b \stackrel{f}{\longrightarrow} b \otimes_{A} 1 & \bmod N\left(B \otimes_{A} B\right) \\
b \stackrel{g}{\longrightarrow} 1 \otimes_{A} b & \bmod N\left(B \otimes_{A} B\right) .
\end{array}
$$

Then (see: section II) we prove that weak normality descends by faithful flatness (see: Proposition (II. 1)), from which we deduce that a noetherian local ring, whose completion is weakly normal, is such.

We then restrict ourselves to noetherian rings and in section III we prove that the property " $A$ is weakly normal in $B$ " is preserved by any reduced base change (see: Theorem (III. 1)).

We apply Theorem (III. 1) to Mori rings to show that reduced homomorphisms with weakly normal generic fibers preserve weak normality.

Received November 20, 1978.

*) This research was supported by GNSAGA of C.N.R. 
So a local excellent ring is weakly normal iff its completion is such (see: Proposition (III.5)). We give also results about the analytic weak normality.

In section IV we show that weak normality is a local property and can be checked at the primes of depth 1 .

We apply this fact in Section V, where we give conditions in order that the product of two weakly normal $k$-schemes is weakly normal and we study the properties of weakly normal morphisms.

In section VI we give two counterexamples.

\section{Preliminaries}

We remark that, if $A$ is a ring, $B$ an overring of $A$ integral over $A$, the seminormalization of $A$ in $B$ is defined being the subring of $B$

$$
{ }_{B}^{+} A=\left\{b \in B / \forall x \in \operatorname{Spec}(A), b_{x} \in A_{x}+R\left(B_{x}\right)\right\}
$$

where $R\left(B_{x}\right)$ is the radical of $B_{x}$; while the weak normalization (or weak integral closure) of $A$ in $B$ is defined being the subring of $B$

$$
{ }_{B}^{*} A=\left\{b \in B / \forall x \in \operatorname{Spec}(A), \exists m \in N:\left(b_{x}\right)^{p^{m}} \in A_{x}+R\left(B_{x}\right)\right\}
$$

where $p$ is the characteristic exponent of the field $k(x)$.

If $A={ }_{B}^{+} A$ we say that $A$ is seminormal (SN for short) in $B$; if $A=$ ${ }_{B}^{*} A$ we say that $A$ is weakly normal (WN for short) in $B$.

Since ${ }_{B}^{+} A$ is contained in ${ }_{B}^{*} A$ (and they coincide when $\operatorname{char} k(x)=0$ ) then if $A$ is WN in $B$, it is also SN in $B$.

The ring ${ }_{B}^{+} A$ is always $\mathrm{SN}$ in $B$ and (see: [15]) it is the largest among the subrings $A^{\prime}$ of $B$ containing $A$ and such that

i) $\forall x \in \operatorname{Spec}(A)$, there exists exactly one $x^{\prime} \in \operatorname{Spec}\left(A^{\prime}\right)$ over $x$ (i.e. such that $j_{x^{\prime}} \cap A=j_{x}$ );

ii) the canonical homomorphism $k(x) \rightarrow k\left(x^{\prime}\right)$ is an isomorphism.

In the same way (see: [1] and [2]) ${ }_{B}^{*} A$ is always $\mathrm{WN}$ in $B$ and it is the largest among the subrings $A^{\prime}$ of $B$ containing $A$ and such that:

i) $\forall x \in \operatorname{Spec}(A)$, there exists exactly one $x^{\prime} \in \operatorname{Spec}\left(A^{\prime}\right)$ over $x$;

ii) the canonical homomorphism $k(x) \rightarrow k\left(x^{\prime}\right)$ makes $k\left(x^{\prime}\right)$ a purely inseparable extension of $k(x)$.

If $B$ is the integral closure of a reduced ring $A$ in its total ring of fractions, then ${ }_{B}^{*} A\left({ }_{B}^{+} A\right)$ is denoted by ${ }^{*} A\left({ }^{+} A\right)$ and if $A={ }^{*} A\left(A={ }^{+} A\right)$ the ring $A$ is said to be weakly normal (seminormal). 
We remark that there are seminormal rings which are not weakly normal. For example, if $k$ is a field of characteristic 2, then the ring $A$ $=k[X, Y, Z] /\left(X Y^{2}-Z^{2}\right)$ is seminormal, while its weak normalization is isomorphic to the ring $k[X, Y]$.

\section{A characterization of weak normalization}

Let $A$ be a ring, $B$ an overring of $A$, integral over $A$. In [11] we have defined

$$
\hat{A}_{B}=\left\{b \in B / b \otimes_{A} 1-1 \otimes_{A} b \in N\left(B \otimes_{A} B\right)\right\}
$$

and we have proved that $\hat{A}_{B}$ is the smallest of all the subrings $A^{\prime}$ of $B$ which contain $A$ and are such that $\left(\hat{A}^{\prime}\right)_{B}=A^{\prime}$.

This fact can be stated as follows:

(I. 1) Proposition.

i) $\hat{A}_{B}$ is a subring of $B$ containing $A$;

ii) if $C$ is any ring such that $A \subseteq C \subseteq B$, then $\hat{A}_{B} \subseteq \hat{C}_{B}$;

iii) $\hat{A}_{B}=\left(\hat{A}_{B}\right)_{B}^{\hat{B}}$;

iv) if $D$ is a ring such that $A \subseteq D \subseteq \hat{A}_{B}$, then $\hat{D}_{B}=\hat{A}_{B}$.

Proof. i) and ii) are clear; iv) is obvious once we have proved iii).

iii) Clearly $\hat{A}_{B} \subseteq\left(\hat{A}_{B}\right)_{B}$. In order to prove the inverse inclusion, we observe that, if $b \in\left(\hat{A}_{B}\right)_{B}$, then $b \otimes_{Z} 1-1 \otimes_{Z} b \in \operatorname{Rad}\left(\operatorname{ker}\left(B \otimes_{Z} B \rightarrow\right.\right.$ $\left.B \otimes_{\hat{A}_{B}} B\right)$ ), hence there exists $m \in N$ such that

$$
\left(b \otimes_{Z} 1-1 \otimes_{Z} b\right)^{m}=\sum_{\substack{\text { finite } \\ b_{i}^{\prime}, b b_{i}^{\prime} \in B \\ \hat{a}_{i} \in \hat{A}_{B}}}\left(b_{i}^{\prime} \otimes_{Z} b_{i}^{\prime \prime}\right)\left(\hat{a}_{i} \otimes_{Z} 1-1 \otimes_{Z} \hat{a}_{i}\right) .
$$

But, as, $\hat{a}_{i} \in \hat{A}_{B}$, for every $i$ there exists $n_{i} \in N$ such that

$$
\left(\hat{a}_{i} \otimes_{Z} 1-1 \otimes_{Z} \hat{a}_{i}\right)^{n_{i}} \in \operatorname{ker}\left(B \otimes_{Z} B \rightarrow B \otimes_{A} B\right)=I,
$$

then

$$
\left(b \otimes_{Z} 1-1 \otimes_{Z} b\right)^{m \Sigma n_{i}} \in I, \quad \text { i.e. } b \in \hat{A}_{B} .
$$

(I. 2) Lemma. Let $A$ be a ring, let $B$ and $C$ be two rings containing $A$ and integral over $A$, and $f: B \rightarrow C$ an integral homomorphism of $A$ algebras such that $\operatorname{ker} f \subseteq N(B)$.

Then the canonical homomorphism

$$
h:\left(B \otimes_{A} B\right)_{\mathrm{red}} \rightarrow\left(C \otimes_{A} C\right)_{\mathrm{red}}
$$


is injective.

Proof. The morphism $f^{*}: \operatorname{Spec}(C) \rightarrow \operatorname{Spec}(B)$ induced by $f$ is surjective, hence (see: [9], par. 1, Proposition (3.6.1)) the morphism

$$
g: \operatorname{Spec}\left(C \otimes_{A} C\right) \rightarrow \operatorname{Spec}\left(B \otimes_{A} B\right)
$$

is surjective. The conclusion follows by [9], par. 1, Corollary (1.2.6).

(I. 3) Proposition. Let $A \subseteq B \subseteq C$ be rings such that $C$ is integral over $A$.

Then $\hat{A}_{C} \cap B=\hat{A}_{B}$.

Proof. By (I. 1) we have $\hat{A}_{B} \subseteq \hat{A}_{C} \cap B$. Conversely, if $b \in \hat{A}_{C} \cap B$, then $b \in B$ and $b \otimes_{A} 1-1 \otimes_{A} b \in N\left(C \otimes_{A} C\right)$, but, by (I. 2), we have that $b \otimes_{A} 1-1 \otimes_{A} b \in N\left(B \otimes_{A} B\right)$, hence $b \in \hat{A}_{B}$.

Applying the above to $A \subseteq \hat{A}_{B} \subseteq B$, we have

(I. 4) Corollary.

$$
\hat{A}_{B}=\hat{A}_{\hat{A}_{B}} \text {. }
$$

Recall the following definition:

(I. 5) Definition. Let $S, T$ be two commutative rings, let $f: S \rightarrow T$ be a ring homomorphism. We say that $T$ is a radical $S$-algebra (via $f$ ) if for every $\mathfrak{B} \in \operatorname{Spec}(S)$ there is at most one $\mathfrak{p} \in \operatorname{Spec}(T)$ such that $f^{-1}(\mathfrak{p})$ $=\mathfrak{P}$ and furthermore for any such pair $\mathfrak{P}, \mathfrak{p}$, the map $S / \mathfrak{R} \rightarrow T / \mathfrak{p}$ induced by $f$ makes the field of fractions of $T / p$ a purely inseparable extension of that of $S / \mathfrak{P}$.

It is equivalent to say any two distinct homomorphisms of $T$ into a field have distinct compositions with $f$; or again $t \otimes_{S} 1-1 \otimes_{S} t \in N\left(T \otimes_{S} T\right)$ for all $t \in T$, i.e. the kernel $H$ of the canonical map $T \otimes_{S} T \rightarrow T$ is a nilideal (see: [9]; p. 246, Proposition (3.7.1)).

(I. 6) THEOREM.

$$
\hat{A}_{B}={ }_{B}^{*} A \text {. }
$$

Proof. By [1] the weak normalization ${ }_{B}^{*} A$ of $A$ in $B$ is a radical $A$ algebra, hence $b \otimes_{A} 1-1 \otimes_{A} b \in N\left({ }_{B}^{*} A \otimes_{A}{ }_{B}^{*} A\right)$ for all $b \in{ }_{B}^{*} A$, therefore $b \otimes_{A} 1-1 \otimes_{A} b \in N\left(B \otimes_{A} B\right)$ for all $b \in{ }_{B}^{*} A$; so ${ }_{B}^{*} A \subseteq \hat{A}_{B}$.

On the other hand, by (I. 4) 


$$
\hat{A}_{B}=\hat{A}_{\hat{A}_{B}}=\left\{c \in \hat{A}_{B} / c \otimes_{A} 1-1 \otimes_{A} c \in N\left(\hat{A}_{B} \otimes_{A} \hat{A}_{B}\right)\right\},
$$

so $\hat{A}_{B}$ is radical over $A$, hence $\hat{A}_{B} \subseteq{ }_{B}^{*} A$, since by [1] and [2], ${ }_{B}^{*} A$ is the greatest radical $A$-algebra between $A$ and $B$.

(I. 7) Remark. Let $f, g$ be the ring homomorphisms

$$
\begin{aligned}
& B \underset{f}{\longrightarrow}\left(B \otimes_{A} B\right)_{\mathrm{red}} \\
& b \stackrel{f}{\longmapsto} b \otimes_{A} 1 \quad \bmod N\left(B \otimes_{A} B\right) \\
& b \stackrel{g}{\longmapsto} 1 \otimes_{A} b \quad \bmod N\left(B \otimes_{A} B\right) .
\end{aligned}
$$

By (I. 6)

$$
{ }_{B}^{*} A=\{b \in B / f(b)=g(b)\}=\operatorname{ker}(f-g)
$$

where $f-g$ is the $A$-module homomorphism

$$
\begin{aligned}
& B \longrightarrow\left(B \otimes_{A} B\right)_{\text {red }} \\
& b \longmapsto b \otimes_{A} 1-1 \otimes_{A} b \quad \bmod N\left(B \otimes_{A} B\right) ;
\end{aligned}
$$

so we can see the weak normalization of $A$ in $B$ as the kernel of the two maps $f, g$ (see: [9], par. 0, (1.4)), that is the sequence

$$
{ }_{B}^{*} A \longrightarrow B \underset{g}{\stackrel{f}{\longrightarrow}}\left(B \otimes_{A} B\right)_{\mathrm{red}}
$$

is exact.

(I. 8) Corollary. Let $A \subseteq B \subseteq C$ be rings such that $C$ is integral over $A$.

If $A$ is $W N$ in $B$ and $B$ is $W N$ in $C$, then $A$ is $W N$ in $C$.

Proof. Observe that ${ }_{C}^{*} A \subseteq B$. In fact, let $c \in{ }_{C}^{*} A$. Then $c \otimes_{A} 1-1$ $\otimes_{A} c \in N\left(C \otimes_{A} C\right)$, hence $c \otimes_{B} 1-1 \otimes_{B} c \in N\left(C \otimes_{B} C\right)$, so $c \in{ }_{C}^{*} B=B$.

By Proposition (I. 3), we have ${ }_{B}^{*} A={ }_{C}^{*} A \cap B$, so ${ }_{B}^{*} A={ }_{C}^{*} A$ and, since $A$ is WN in $B,{ }_{c}^{*} A=A$.

\section{Faithfully flat descent}

In this section we prove that weak normality descends by faithful flatness; so a noetherian local ring, whose completion is weakly normal, is such.

(II. 1) Proposition (Faithfully flat descent). Let $f: A \rightarrow A^{\prime}$ be a faithfully flat ring homomorphism. Put $B^{\prime}=A^{\prime} \otimes_{A} B$. If $A^{\prime}$ is weakly normal 
in $B^{\prime}$, then $A$ is weakly normal in $B$.

Proof. We first observe that if we consider the commutative diagram

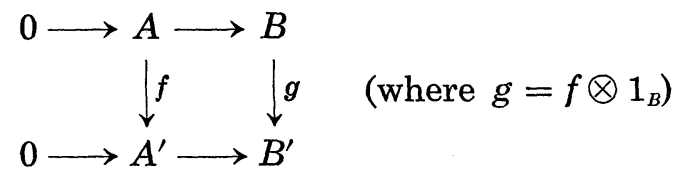

by (I. 6) we have that $g\left({ }_{B}^{*} A\right) \subseteq{ }_{B^{\prime}}^{*} A^{\prime}$, hence

$$
{ }_{B}^{*} A \subseteq g^{-1}\left(g\left({ }_{B}^{*} A\right)\right) \subseteq g^{-1}\left({ }_{B}^{*} A^{\prime}\right) .
$$

Besides, by (I. 1) i), $A \subseteq{ }_{B}^{*} A$, and by assumption $A^{\prime}={ }_{B^{\prime}}^{*} A^{\prime}$, therefore

$$
A \subseteq{ }_{B}^{*} A \subseteq g^{-1}\left(g\left({ }_{B}^{*} A\right)\right) \subseteq g^{-1}\left({ }_{B}^{*} A^{\prime}\right)=g^{-1}\left(A^{\prime}\right) .
$$

We observe that, since $A \subseteq g^{-1}\left(A^{\prime}\right)$, the homomorphism $g$ induces an $A$ module homomorphism $\bar{g}: B / A \rightarrow B^{\prime} \mid A^{\prime}$. Now let us consider the commutative diagram

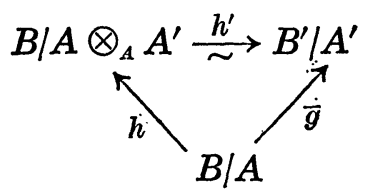

Since $h^{\prime}$ is an isomorphism and $h$ is injective as $A^{\prime}$ is faithfully flat over $A$ (see: [3], ch. 1, p. 50, Proposition 8) we have that $\bar{g}$ is injective, that is $g^{-1}\left(A^{\prime}\right)=A$. The conclusion follows by (1).

(II. 2) Conollary. Let $A$ be a ring, let $f: A \rightarrow A^{\prime}$ be a faithfully flat ring homomorphism.

Then $A$ is $W N$ if $A^{\prime}$ is such.

Proof. Since $A$ is reduced and $A^{\prime}$ is flat over $A$, then $A^{\prime}$ and $A^{\prime} \otimes_{A} \bar{A}$ have the same total ring of fractions, hence $A^{\prime} \otimes_{A} \bar{A} \subseteq \bar{A}^{\prime}$.

By assumption $A^{\prime}$ is weakly normal, then, by (I. 3) ${ }_{\left(A^{\prime} \otimes_{A} \frac{*}{A}\right)}\left(A^{\prime}\right)=A^{\prime}$, thus $A$ is WN by (II. 1).

(II. 3) CoRollary. Let $A$ be a local noetherian ring. If the completion of $A$ is weakly normal, then $A$ is such.

Later on we always consider noetherian rings. 


\section{Permanence of weak normalization}

In this section we prove that the property " $A$ is $\mathrm{WN}$ in $B$ " is stable under reduced base changes. We then apply this fact to prove that reduced homomorphisms having normal generic fibers (e.g. normal homomorphisms) preserve weak normality, and to show that a local excellent ring is $\mathrm{WN}$ iff its completion is such.

Recall that a ring homomorphism $h: A \rightarrow C$ is reduced (resp.: normal, weakly normal, etc.) if it is flat and its fibers are geometrically reduced (resp.: normal, WN, etc.) ${ }^{(1)}$.

We use such language, employed by Grothendieck in [10] and Matsumura in [13], even if it is a little different from the one of [1] (see: Section 5).

(III. 1) Theorem. Let $B$ be a finite overring of $A$, and let $h: A \rightarrow C$ be a reduced ring homomorphism.

Then $\left({ }_{B}^{*} A\right) \otimes_{A} C={ }_{\left(B \otimes_{A} C\right)}^{*}\left(A \otimes_{A} C\right)$.

Proof. By (I. 7), the sequence

$$
{ }_{B}^{*} A \longrightarrow B \Longrightarrow\left(B \otimes_{A} B\right)_{\text {red }}
$$

is exact; $C$ is flat over $A$, hence

$$
\left({ }_{B}^{*} A\right) \otimes_{A} C \longrightarrow B \otimes_{A} C \Longrightarrow\left(B \otimes_{A} B\right)_{\mathrm{red}} \otimes_{A} C
$$

is exact. On the other hand, being $h: A \rightarrow C$ a reduced homomorphism $\left(B \otimes_{A} B\right)_{\text {red }} \otimes_{A} C$ is reduced (see: [13]), hence

$$
\begin{aligned}
\left(B \otimes_{A} B\right)_{\mathrm{red}} \otimes_{A} C & =\left[\left(B \otimes_{A} B\right)_{\mathrm{red}} \otimes_{A} C\right]_{\mathrm{red}} \\
& =\left[\left(B \otimes_{A} B\right) \otimes_{A} C\right]_{\mathrm{red}} \\
& =\left(\left(B \otimes_{A} C\right) \otimes_{\left(A \otimes_{A} C\right)}\left(B \otimes_{A} C\right)\right)_{\mathrm{red}} .
\end{aligned}
$$

Therefore

$$
\left({ }_{B}^{*} A\right) \otimes_{A} C \longrightarrow B \otimes_{A} C \longrightarrow\left(\left(B \otimes_{A} C\right) \otimes_{\left(A \otimes_{A} O\right)}\left(B \otimes_{A} C\right)\right)_{\mathrm{red}}
$$

is exact. The conclusion follows by (I.7).

(III. 2) Corollary. Let $A, B, C$ as in (III. 1). If $A$ is weakly normal in $B$, then $A \otimes_{A} C=C$ is weakly normal in $B \otimes_{A} C$ and the converse is true if $C$ is faithfully flat over $A$ (see also (II. 1)).

(1) That is $\forall \Re \in \operatorname{Spec}(A),\left(k(\Re) \otimes_{A} C\right) \otimes_{k(\mathfrak{B})} K$ is reduced (resp.: normal, WN, etc.) for every finitely generated extension $K$ of the field $k(\mathfrak{B})$. 
Proof. Since $A={ }_{B}^{*} A$, then ${ }_{\left(B \otimes_{A} C\right)}^{*}\left(A \otimes_{A} C\right)=\left({ }_{B}^{*} A\right) \otimes_{A} C=A \otimes_{A} C$.

Besides, if $C$ is faithfully flat over $A$, then $A \otimes_{A} C=\left({ }_{B}^{*} A\right) \otimes_{A} C$ implies $A={ }_{B}^{*} A$.

Later on we always suppose $A$ is a Mori ring, that is $A$ is reduced and its integral closure $\bar{A}$ (in its total ring of fractions) is finite over $A$.

(III. 3) Proposition. Let $h: A \rightarrow C$ be a reduced ring homomorphism having normal generic fibers (e.g. $h$ is normal).

Then:

i) $\left({ }^{*} A\right) \otimes_{A} C={ }^{*} C$;

ii) if $A$ is $W N$, then $C$ is $W N$ (and the converse holds if $h$ is faithfully flat).

Proof. By [7], (3.1) we have $\bar{C}=\bar{A} \otimes_{A} C$. The conclusion follows by (III. 1) and (III. 2).

(III. 4) Corollary. Let $A$ be a local excellent ring. Then $A$ is $W N$ if and only if its completion is WN.

Proof. It follows by (II. 3) and (III. 3), since $A \rightarrow \hat{A}$ is normal (see: [13], Theorem 79).

(III. 5) Proposition. Let $A$ be a local excellent ring, $B$ a reduced local ring, $f: A \rightarrow B$ a local homomorphism. Assume $A$ and $B$ have the same completion. Then $A$ is $W N$ if and only if $B$ is such.

Proof. Since $B$ has the same completion as $A$, then $B$ is faithfully flat over $A$. The conclusion follows from (II. 2) and (III. 4).

(III. 6) Corollary. The local ring $A$ at a point $x$ of a complex algebraic variety $V$ is $W N$ iff the local ring $B$ at $x$ of the corresponding analytic space is such.

Proof. It follows by (III. 5), since $A$ is excellent and $B$ is noetherian and has the same completion as $A$ (see: [14]).

Remark. The above result is the same of [8], Corollary (5.4), but it is obtained by simpler methods. In fact, it is based on Theorem (III. 1), which is proved by standard results of commutative algebra, while the analogue theorem of Greco and Traverso is obtained using Traverso's gluings. 
(III. 7) Proposition. Let $A$ be a ring, let $C=A[[x]]$. Then $A$ is $W N$ iff $C$ is such.

Moreover, if we assume $A$ is a Zariski universally japanese ring (i.e. a Nagata ring, see: [11]), then $A$ is $W N$ iff the ring $A\left\{x_{1}, \cdots, x_{n}\right\}$ of restricted formal series in a finite number of indeterminates is such.

Proof. Since $C$ is faithful flat over $A$, then, if $C$ is WN, $A$ is such by (II. 3). The converse results by (III. 3) and [8], Lemma (5.6).

The second part follows by (III. 3) and [12] (see: Proposition 7).

\section{Weak normality as local property}

In this section we show that weak normality is a local property and can be checked at the primes of depth 1 .

The results are quite similar to Greco and Traverso's ones and the proofs too.

(IV. 1) Proposition. Let $B$ be a finite overring of $A$. Put $C={ }_{B}^{*} A$. Let $S$ be a multiplicative subset of $A$. Then:

i) $S^{-1} C$ is the weak normalization of $S^{-1} A$ in $S^{-1} B$;

ii) if $A$ is $W N$ in $B$, then $S^{-1} A$ is $W N$ in $S^{-1} B$.

Proof. It follows from (III. 1) and (III. 2).

(IV.2) Corollary. Let $A$ be a ring; let $S$ be a multiplicative subset of $A$. Then if $A$ is $W N$, then $S^{-1} A$ is $W N$.

Moreover, if $A$ is a Mori ring, and $C={ }^{*} A$, then $*\left(S^{-1} A\right)=S^{-1} C$.

(IV.3) Theorem. Let $B$ be a finite overring of $A$. The following statements are equivalent:

i) $A$ is $W N$ in $B$;

ii) $A_{\mathfrak{P}}$ is $W N$ in $B_{\mathfrak{P}} \quad \forall \mathfrak{P} \in \operatorname{Spec}(A)$;

iii) $A_{\mathfrak{m}}$ is $W N$ in $B_{\mathrm{m}} \quad \forall \mathrm{m} \in \operatorname{Specm}(A)$;

iv) $A_{\Re}$ is $W N$ in $B_{\Re} \quad \forall \Re \in \operatorname{Ass}(B / A)$.

Besides, if every regular element of $A$ is regular in $B$, the above are equivalent to:

v) $A_{\Re}$ is $W N$ in $B_{\mathfrak{\beta}}$ whenever $\operatorname{depth}\left(A_{\mathfrak{\beta}}\right)=1$.

If moreover $A$ is $S_{2}$ the above are equivalent to:

vi) $A_{\Re}$ is $W N$ in $B_{\Re}$ whenever $h t \Re=1$. 
Proof. We show iii) implies i); the remainder follows from (IV. 1) with an argument similar to Theorem (2.6) of [8].

By (IV. 1) we have $\left({ }_{B}^{*} A\right)_{\mathfrak{m}}={ }_{B_{\mathfrak{m}}}^{*}\left(A_{\mathrm{m}}\right)$, hence $\left({ }_{B}^{*} A\right)_{\mathfrak{m}}=A_{\mathfrak{m}}$ for each $\mathfrak{m} \in \operatorname{Specm}(A)$. Then $\left({ }_{B}^{*} A / A\right) \otimes_{A} A_{\mathfrak{m}}=0$ for each $\mathfrak{m} \in \operatorname{Specm}(A)$, hence ${ }_{B}^{*} A=A$.

(IV. 4) Corollary. Let $A$ be a Mori ring, let $\bar{A}$ be its integral closure. Then the following are equivalent:

i) $A$ is $W N$;

ii) $A_{\mathfrak{B}}$ is $W N \quad \forall \Re \in \operatorname{Spec}(A)$;

iii) $A_{\mathrm{m}}$ is $W N \quad \forall \mathfrak{m} \in \operatorname{Specm}(A)$;

iv) $A_{\Re}$ is $W N \quad \forall \Re \in \operatorname{Ass}(\bar{A} / A)$;

v) $A_{\mathfrak{\beta}}$ is $W N \quad$ whenever $\operatorname{depth}\left(A_{\mathfrak{\beta}}\right)=1$.

If moreover $A$ is $S_{2}$ the above are equivalent to

vi) $A_{\Re}$ is $W N \quad$ whenever $\operatorname{dim} A_{\Re}=1$.

\section{Weakly normal morphisms and products}

In this section we prove that under suitable assumptions the product of two WN $k$-schemes is WN. This is used to study the properties of WN morphisms, answering in particular to a question raised by Andreotti and Bombieri on the product of two WN morphisms (see: [1], par. 5).

All schemes we consider are assumed to be locally noetherian.

(V.1) Proposition. Let $f: A \rightarrow B$ be a reduced homomorphism and assume $A$ is weakly normal.

Then $B$ is weakly normal iff $B$ is Mori and the generic fibers of $f$ are weakly normal.

Proof. It is quite similar to [8], Theorem (5.8) using the results of section IV and (I. 8).

(V.2) THeOREM. Let $k$ be a field, let $X, Y$ be two $k$-schemes, such that $X$ is locally essentially of finite type over $k$.

If $X$ is weakly normal and $Y$ is geometrically weakly normal, then $X \times_{k} Y$ is geometrically weakly normal.

Proof. The question is local, hence we may assume $X=\operatorname{Spec}(A)$, $Y=\operatorname{Spec}(B)$ where $A$ is essentially of finite type over $k$.

Let $K / k$ be a finitely generated field extension of $k$. We have to show 
that $\left(A \otimes_{k} B\right) \otimes_{k} K=A \otimes_{k}\left(B \otimes_{k} K\right)$ is WN. But $B \otimes_{k} K$ is geometrically WN and then, after replacing $B$ with $B \otimes_{k} K$ it is sufficient to show that $A \otimes_{k} B$ is WN.

We can observe that the canonical map $k \rightarrow B$ is reduced (since $B$ is geometrically WN), then the same holds for $h: A \rightarrow A \otimes_{k} B$ (see: [10], part II, (6.8) and (7.3)). Moreover the fibers of $h$ are WN. Indeed, for each $\mathfrak{P} \in \operatorname{Spec}(A)$ we have $k(\mathfrak{P}) \otimes_{A}\left(A \otimes_{k} B\right)=k(\mathfrak{P}) \otimes_{A} B$ which is WN as $B$ is geometrically WN and $k(\mathfrak{P})$ is a finitely generated extension of $k$ (since $\mathrm{A}$ is essentially of finite type over $k$ ). The conclusion follows by (V.1) since $A \otimes_{k} B$ is a Mori ring (see: [8], Corollary (5.9)).

Remark. Theorem (V.2) is based on Proposition (V.1) which is an obvious generalization of Theorem (5.8) of [8], however no assumptions are made on the field $k$, while in [8], Corollary (5.9) it is supposed to be perfect.

(V. 3) Corollary. Let $X$ be a WN k-scheme, $K$ a separable field extension of the field $k$. Assume either $X$ or $K$ is essentially of finite type over $k$.

Then $X \times{ }_{k} K$ is $W N$.

(V.4) Corollary. Let $k$ be a perfect field (e.g. algebraically closed), let $X$ and $Y$ be two WN k-schemes, one of which is locally essentially of finite type over $k$.

Then $X \times_{k} Y$ is weakly normal.

(V.5) Lemma. Let $C$ be a ring, let $A, B, A^{\prime}$ be three $C$-algebras essentially of finite type over $C$. Let $f: A \rightarrow B$ be a WN C-morphism.

Then the morphism $A \otimes_{C} A^{\prime} \rightarrow B \otimes_{C} A^{\prime}$ is WN.

Proof. Let $\mathfrak{p} \in \operatorname{Spec}(A)$. If $\mathfrak{R}=j^{-1}(\mathfrak{p})$ (where $j$ is the canonical map $\left.A \rightarrow A \otimes_{C} A^{\prime}\right), k(\mathfrak{p})$ is finitely generated over $k(\mathfrak{R})$, moreover

$$
\begin{aligned}
k(\mathfrak{p}) \otimes_{\left(A \otimes_{C} A^{\prime}\right)}\left(B \otimes_{C} A^{\prime}\right) & =k(\mathfrak{p}) \otimes_{\left(A \otimes_{C} A^{\prime}\right)}\left(B \otimes_{A}\left(A \otimes_{C} A^{\prime}\right)\right) \\
& =k(\mathfrak{p}) \otimes_{A} B=k(\mathfrak{p}) \otimes_{k(\mathfrak{P})}\left(k(\mathfrak{P}) \otimes_{A} B\right)
\end{aligned}
$$

which is geometrically WN, since $k(\mathfrak{P}) \otimes_{A} B$ is geometrically WN.

(V. 6) Proposition. Let $f: X \rightarrow Y, g: Y \rightarrow Z$ be two weakly normal morphisms of schemes.

Then $g \circ f$ is $W N$. 
Proof. Assume $Z=\operatorname{Spec}(A), Y=\operatorname{Spec}(B), X=\operatorname{Spec}(C)$. For each $\mathfrak{P} \in \operatorname{Spec}(A)$ we have $k(\mathfrak{P}) \otimes_{A} C=\left(k(\mathfrak{P}) \otimes_{A} B\right) \otimes_{B} C$. The morphism $f^{*}: B$ $\rightarrow C$ is WN and $k(\mathfrak{R}) \otimes_{A} B$ is WN and essentially of finite type over $B$, therefore the morphism $k(\mathfrak{P}) \otimes_{A} B \rightarrow\left(k(\mathfrak{P}) \otimes_{A} B\right) \otimes_{B} C$ is WN by (V. 5). So by (V. 1) $k(\mathfrak{P}) \otimes_{A} C$ is WN.

Moreover, if we consider the morphism

$$
\left(k(\mathfrak{P}) \otimes_{A} B\right) \otimes_{k(\mathfrak{P})} K \rightarrow\left(k(\mathfrak{P}) \otimes_{A} C\right) \otimes_{k(\mathfrak{P})} K
$$

where $K$ is a finitely generated extension of $k(\mathfrak{P})$, with the same arguments used above we can prove that $\left(k(\mathfrak{P}) \otimes_{A} C\right) \otimes_{k(\mathfrak{R})} K$ is WN, so $k(\mathfrak{P}) \otimes_{A} C$ is geometrically WN.

The following theorem answers to a question raised in [1] (see: par. 5).

(V. 7) Theorem. Let $f: X \rightarrow Y, g: X^{\prime} \rightarrow Y^{\prime}$ be two weakly normal morphisms of S-schemes. Assume $X^{\prime}, Y$ be locally essentially of finite type.

Then the morphism $f \times g: X \times{ }_{s} X^{\prime} \rightarrow Y \times{ }_{s} Y^{\prime}$ is WN.

Proof. We may assume $Y=\operatorname{Spec}(A), X=\operatorname{Spec}(B), Y^{\prime}=\operatorname{Spec}\left(A^{\prime}\right)$, $X^{\prime}=\operatorname{Spec}\left(B^{\prime}\right)$ where $A^{\prime}, B$ are essentially of finite type over $C$ (where $\operatorname{Spec}(C)=S)$. If $f^{*}: A \rightarrow B, g^{*}: A^{\prime} \rightarrow B^{\prime}$ are the morphisms induced by $f, g$ respectively, then the morphism

$$
f^{*} \otimes_{C} g^{*}: A \otimes_{C} A^{\prime} \rightarrow B \otimes_{C} B^{\prime}
$$

can be written as $f^{*} \otimes g^{*}:=\left(1_{B} \otimes g^{*}\right) \circ\left(f^{*} \otimes 1_{A^{\prime}}\right)$ by the commutativity of the diagram

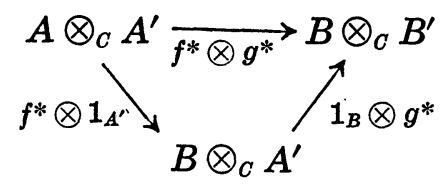

The conclusion follows by (V.5) and (V.6).

(V. 8) Remark. We can observe that WN morphisms are $P$-morphisms according to [10], part II, (7.3.1). Indeed by the definitions and by (V.6) and (II. 1), it follows that WN morphisms satisfies axioms $P_{\mathrm{I}}^{\prime}, P_{\mathrm{II}}, P_{\mathrm{III}}, P_{\mathrm{IV}}$.

(V.9) Remark. The results of this section hold also for seminormality. In fact, after replacing "weakly normal" with "seminormal", all the propositions of this section can be proved by the same arguments. 


\section{Two counterexamples}

In this section we give an example of a normal local ring whose completion is not seminormal (hence not weakly normal) and an example of a geometrically reduced ring which is weakly normal but not geometrically weakly normal.

(VI. 1) Example. Let $k$ be a field such that char $k \neq 2$. Let $X, Y$ be analytically independent over the field $k$ and let $f=Y+o(2) \in k[X][[Y]]$ be transcendental over $k(X, Y)$.

Let $R=k\left(X, Y, f^{3}\right) \cap k[[X, Y]]$ and let $A=\left(R[Z] /\left(Z^{2}-f^{3}\right)\right)_{(x, y, Z)}$. By [16], Propositions 3 and 7 (or [6], Proposition 4.2) we have that $R$ is a regular local ring of dimension 2 and that $f^{3}$ is prime in $R$, then $A$ is a normal local ring (see: [5], p. 49, Lemma (11.1)). Moreover, again by [16]

$$
\begin{aligned}
\hat{A} & =A \otimes_{R} \hat{R}=\hat{R}[Z] /\left(Z^{2}-f^{3}\right)=k[[X, Y]][Z] /\left(Z^{2}-f^{3}\right) \\
& =k[[X, Y, Z]] /\left(Z^{2}-f^{3}\right) .
\end{aligned}
$$

Since $B=\hat{A}_{(z, f)} \simeq k[[X, Y, Z]]_{(Y, Z)} /\left(Z^{2}-Y^{3}\right)$ is not seminormal as $\operatorname{Gr}(B)$ is not reduced (cf.: [4]), $\hat{A}$ is not seminormal by [8], Corollary (2.7) (hence it is not weakly normal).

(VI. 2) Example. Let $k$ be a field of characteristic $p(p>2)$. Consider $A=k[x, y]=k[X, Y] /\left(X^{2}+Y^{p}-a\right)$ where $a \in k, \sqrt[p]{a} \notin k$.

$A$ is a one dimensional domain which is normal, hence weakly normal. Indeed, let $\bar{k}$ be an algebraic closure of $k$, let

$$
\bar{A}=A \otimes_{k} \bar{k}=\bar{k}[X, Y] /\left(X^{2}+Y^{p}-a\right) .
$$

By the jacobian criterion, the unique maximal ideal $\mathfrak{m}$ of $A$ such that $A_{\mathrm{m}}$ could not be regular is the contraction to $A$ of the maximal ideal of $\bar{A}$ $\overline{\mathfrak{m}}=(x, y-b)$ with $b \in \bar{k}$ such that $b^{p}=a$. But

$$
\begin{aligned}
\mathfrak{m} & =\overline{\mathfrak{m}} \cap A=\left(x, y^{p}-a\right)=\left(X, Y^{p}-a\right) /\left(X^{2}+Y^{p}-a\right) \\
& =\left(X, X^{2}+Y^{p}-a\right) /\left(X^{2}+Y^{p}-a\right)=(x)
\end{aligned}
$$

hence $A_{m}$ is a $D V R$, then $A$ is normal.

Let $k^{\prime}=k(b)$, and let $A^{\prime}=k^{\prime} \otimes_{k} A=k^{\prime}[X, Y] /\left(X^{2}+(Y-b)^{p}\right) . \quad \mathrm{A}^{\prime}$ is a domain (hence $A$ is geometrically reduced by [10], part II, (6.7.7)), but it is not weakly normal. In fact $k^{\prime}[X, Y] /\left(X^{2}+(Y-b)^{p}\right) \simeq k^{\prime}\left[t^{2}, t^{p}\right]$ and the weak normalization of $k^{\prime}\left[t^{2}, t^{p}\right]$ is $k^{\prime}[t]$ by (I. 6), since 


$$
\left(t \otimes_{k^{\prime}\left[t^{2}, t^{p}\right]} 1-1 \otimes_{k^{\prime}\left[t^{2}, t^{p}\right]} t\right)^{p}=0
$$

hence

$$
t \otimes 1-1 \otimes t \in N\left(k^{\prime}[t] \otimes_{k^{\prime}\left[t^{2}, t^{p}\right]} k^{\prime}[t]\right) .
$$

One can show that $A^{\prime}$ is also not seminormal, by using [4] as in (VI. 1).

\section{REFERENCES}

[1] A. Andreotti and E. Bombieri, Sugli omeomorfismi delle varietà algebriche. Ann. Scuola Norm. Sup. Pisa 23 (1969), 430-450.

[2] E. Bombieri, Seminormalità e singolarità ordinarie. Symp. Math. XI (1973), 205210.

[ 3 ] N. Bourbaki, Algèbre Commutative. Hermann, Paris, 1961.

[4] E. Davis, On the geometric interpretation of seminormality. Proc. Amer. Math. Soc. 68 (1978), 1-5.

[5] R. Fossum, The divisor class group of a Krull domain. Erg. der Math., b. 74, Springer Verlag, 1973.

[6] S. Greco, Two theorems on excellent rings. Nagoya Math. J. 60 (1976), 139-149.

[ 7 ] S. Greco and N. Sankaran, On the separable and algebraic closedness of a Hensel couple in its completion. J. of Algebra 39 (1976), 335-348.

[ 8 ] S. Greco and C. Traverso, On seminormal schemes, to appear on Compositio Math.

[9] A. Grothendieck and J. Dieudonné, Eléments de Géométrie Algébrique I, Grund. der Math. b. 166, Springer Verlag, 1971.

[10] A. Grothendieck, Eléments de Géométrie Algébrique IV, Publ. Math. I.H.E.S. n. 24, Paris 1965.

[11] M. Manaresi, Una caratterizzazione della seminormalizzazione, Boll. Un. Mat. Ital, (5) 15-A (1978), 205-213.

[12] J. Marot, Sur les anneaux de séries formelles restreintes, to appear.

[13] H. Matsumura, Commutative Algebra, W.A. Benjamin Inc., New York, 1970.

[14] J. P. Serre, Géométrie Algébrique et Géométrie Analytique, Ann. Ist. Fourier 6 (1955), 1-42.

[15] C. Traverso, Seminormality and Picard group, Ann. Scuola Norm. Sup. Pisa 24 (1970), 585-595.

[16] P. Valabrega, On two-dimensional regular local rings and a lifting problem, Ann. Scuola Norm. Sup. Pisa 27 (1973), 787-807.

Istituto di Geometria

dell'Università di Bologna

P. za di Porta S. Donato 5

40100 Bologna

Italy 